\title{
Technical considerations in the design of core habitat patches in forest management: A case study using the Patchworks spatial model
}

\author{
by Tom Moore ${ }^{1,2}$ and Greg Tink
}

\begin{abstract}
Incorporation of core area requirements into forest management plans is a requirement in many provinces and for many forest certification systems. The purpose of this paper is to consider how core requirements might be addressed using spatial models and mapping tools, and to assess how well these tools work in practice. To meet these objectives, we applied the spatial model Patchworks to real data representing a portion of a very large forest management unit in northern Ontario, the Gordon Cosens Forest. Three different sets of simulations were conducted to explore the ability of the spatial model to identify core areas, to identify technical challenges, and the means to resolve them.
\end{abstract}

Key words: core area, forest management planning, Patchworks, spatial modelling

\section{RÉSUMÉ}

Linclusion des principales fonctions d'un territoire au sein des plans d'aménagement forestier constitue une obligation édictée par plusieurs provinces et par de nombreux systèmes de certification forestière. L’objectif de cet article est détudier comment les principales fonctions pourraient être traitées au moyen de modèles spatiaux et doutils cartographiques et dévaluer comment ces outils se comportent dans la réalité. Pour atteindre ces objectifs, nous avons utilisé le modèle spatial Patchworks avec les données réelles provenant d'une portion d'une grande unité d’aménagement forestier du nord-est de l'Ontario, la forêt Gordon Cosens. Trois différentes séries de simulation ont été entreprises afin d'explorer la capacité du modèle spatial d'identifier les principales fonctions, les défis techniques et les moyens des résoudre.

Mots clés : fonction principale, planification de laménagement forestier, Patchworks, modélisation spatiale

\section{Introduction}

Concerns about the influence of habitat fragmentation on the conservation of biological diversity have inspired requirements for "core areas" in forest management planning (Eng 1998, Lindenmayer and Franklin 2002). For example, the Ontario Ministry of Natural Resources (OMNR) Forest Management Guidelines for the Provision of Marten Habitat (OMNR 1996) require that:

"Suitable marten habitat should be arranged in "core habitat areas" between 30 and $50 \mathrm{~km}^{2}$ in size. A minimum of 75 percent of core habitat areas should be comprised of suitable stands."

The OMNR also uses a core area concept in its definition of forest disturbances under the Forest Management Guide for Natural Disturbance Pattern Emulation (OMNR 2001), in the caribou mosaic blocks identified according to the Forest Management Guidelines for the Conservation of Woodland Caribou: a Landscape Approach (Racey et al. 1999), and in the Areas of Concern (AOCs) identified for protection of redshouldered hawk nests (OMNR 1998, section 10.2.5). Forest certification systems may also have core area requirements. For example, the Forest Stewardship Council (FSC) National Boreal Standard (FSC 2004) states:

"...large areas (thousands of hectares) of contiguous core forest habitat, representative of the habitat types of the landbase, exist and are maintained in the manage- ment unit. Large cores consist primarily of mature and old forest, but may also contain inclusions of up to $5 \%$ recently disturbed forest. To the greatest extent possible within the current forest condition, large cores do not contain roads and other linear disturbances. In planning future cores, the applicant chooses areas with a high probability of achieving the desired condition (e.g., areas likely to be in a contiguous, roadless condition) and is working within its sphere of influence to achieve this condition (e.g., access management, decommissioning roads, bridge removal, etc.)."

The forest management plan planning process in many provinces requires the documented consideration of the location and abundance of core areas over lengthy planning horizons. Although some cores are static and relatively small (e.g., red-shouldered hawk AOCs), others may be very large (3000 to 10000 or more hectares) and move across the landscape over time, providing that spatial and temporal criteria are met (e.g., caribou mosaic blocks, marten cores, FSC core areas). Some patches must not only meet spatial requirements, but must follow a pre-defined size distribution as well (e.g., disturbance patches; OMNR 2001). Most cores have composition requirements. Criteria for size, shape, period of retention, and composition together present numerous technical challenges for implementation in the context of forest management planning, particularly because such patches are just

\footnotetext{
${ }^{1}$ Spatial Planning Systems, Box 1389, Deep River, Ontario K0J 1P0.

${ }^{2}$ Corresponding author: E-mail: tmoore@spatial.ca
} 
a subset of the requirements that must be met in a typical management plan (e.g., OMNR 2004).

Clearly, the term "core area" as used in forest management is a concept that has a different meaning depending on the context in which it is being used, as well as the agency involved. Habitat biologists may have been the first to coin the term, and for them core area refers to forest interior, which is defined as the area of forest, free of edge effects, where the edge effect is a function of the state of the surrounding habitat (Zipperer 1993). Fig. 1 illustrates a typical definition of "core area" in which the amount of forest interior habitat is important, where forest interior is that portion that is set back from the perimeter of the preferred forest type.

To further complicate the issue of identifying and mapping cores, limited interspersion of other forest or non-forest types may be permitted. In this context, the total acceptable core area may be all land within a perimeter that defines a boundary of an event or specific features. This type of core area includes both the specified type, as well as all of the other areas that are interior inclusions or interspersions of other types (see Fig. 2). For example, in the FSC definition above, the effect that natural, non-commercial vegetative or non-forest types may have on the extent and validity of core composition are not mentioned. How much water, rocky outcrops, muskeg and wetlands are allowed as inclusions within such a core? Little guidance is provided with respect to the desired or allowed shape of the boundary of the core. Are dendritic appendages permitted? What are the size limits for connective corridors?

Finally, the temporal dimension in forest development and management imposes a significant additional layer of complexity for planners. Even disregarding natural disturbance events, core shapes will change over time as stands grow and develop. Harvest activities have long-term impacts on core shape and location, and if sustainability is required then planners must consider how harvest layouts and core areas fit together over many decades.

The most common tool applied in Canadian forest management planning is the aspatial wood supply model. With automated aspatial models, targets can be adjusted to maintain a minimum amount of habitat area throughout the course of the modelling time horizon. However, the spatial arrangement of the habitat into core areas cannot be influenced other than in a limited way by partitioning the data set in to zones.

The typical approach used to address core requirements is to predefine the location of cores over time and incorporate them into the model as deferrals from harvest. The shortcoming of this approach is that predefining core areas precludes the possibility of finding better solutions when other forest management objectives are factored into the decision-making process. As a result, planners cannot be certain that their deferral locations do not unnecessarily restrict or drive plan outcomes. Planning teams could try a number of alternate designs, but the temporal dimension (locating an adequate supply of cores through time) complicates the problem significantly, making it time-consuming to define even a single set of core locations. Clearly an automated method is desirable.

Spatial forest management modelling is a recent technology that simultaneously considers arrangement and conventional wood supply issues. Patchworks is a spatial model

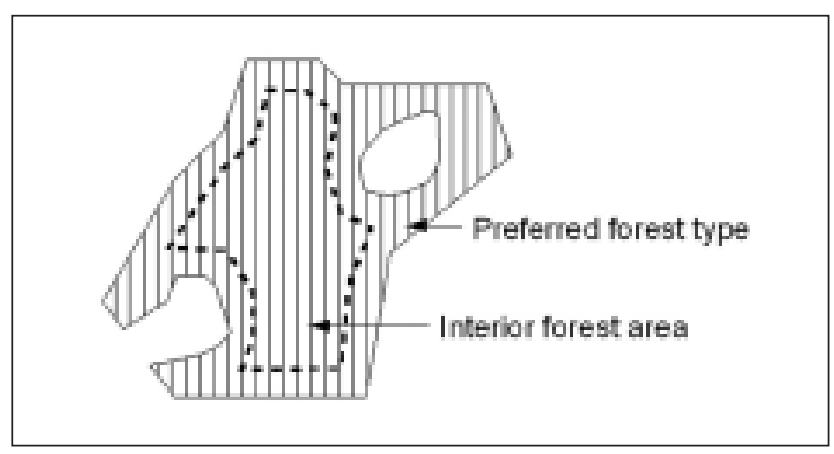

Fig. 1. Interior forest area is defined as the area that is free from edge effects. In this example the dashed line represents the interior of the vertically hatched area.

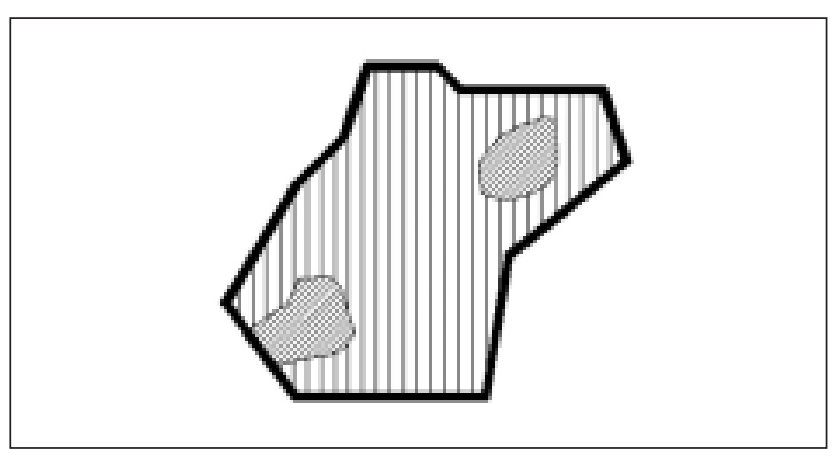

Fig. 2. Core areas represent the featured vegetation type (vertical hatch) as well as inclusions and interspersions or non-featured types (diagonal hatch). The thick black line shows the event boundary of this core. Core boundary locations and their definitions vary, and in Ontario are guideline-specific.

designed for large forest data sets that can be used to measure and track the contiguous area of any given forest condition. Patches can be identified and categorized by size class, and patch characteristics such as the frequency, area, proportion of total, and average shape (as measured by an edge-to-area ratio) for any set of size class categories. In addition to GISlike tools to display spatial and temporal patterns of forest development, the Patchworks program implements a stochastic meta-heuristic-based "solver" that can be used to generate harvest treatment schedules that meet a range of management objectives (Lockwood and Moore 1993). The patch size controls can be expressed as part of a goal programming objective function, and in this way the solver (sometimes referred to as a scheduler) can be used to control the development of patch characteristics over time simultaneously with other traditional forest management objectives.

Although the Patchworks model is useful for identifying and designing contiguous patch shapes on a landscape, its definition of "patch" differs from what we defined above as "core area." Similar to other spatial models that address contiguous area problems (Öhman and Eriksson 1998, Liu et al. 2000), patches measured in Patchworks include only the preferred forest cover, and not interspersions. In a related problem some studies have calculated the influence of adjacent stand condition on interior core area measures (Öhman and 
Eriksson 1998, Wei and Hoganson 2007), but these algorithms require large data structures that limit their application to smaller numbers of polygons.

The Patchworks model contains a tool that can be used to identify and measure core area with interspersions, but it may take up to several minutes to perform an assessment over large areas, and is too slow to be used by the solver (which undertakes thousands of assessments per second). Consequently, automated control of pattern within the solver is restricted to area by patch size class. As with aspatial models we could predefine the location of cores and incorporate these areas into the spatial models as deferrals, but this would not ensure that inferior core locations had been chosen.

\section{Objectives}

Spatial models can measure and design landscape shapes in a fashion, but are the modelling abstractions sufficiently realistic to portray guidelines as they are being proposed by biologists? The purpose of this paper is to consider how core requirements might be addressed using spatial models and mapping tools, and to assess how well these tools work in practice. As we do so we will identify technical issues associated with the identification of core areas, issues that should be considered by biologists, certifiers, and regulators seeking to define core requirements in the most meaningful way possible in the future. To meet these objectives, we applied the spatial model Patchworks to real data representing a portion of a very large forest management unit in northern Ontario, the Gordon Cosens Forest. A number of factors made this a challenging problem and provided a series of questions to be answered during the course of this study:

1) How do neighbour proximity distance and riparian inclusion effect patch size?

The implicit intent in many core area guidelines is that the shape of the core be compact. Cores with large amounts of interspersion or dendritic core shapes with thin corridors may not provide the appropriate level of connectivity or protection of interior. The Patchworks spatial model builds patches by connecting neighbouring polygons provided in the GIS data set. We explored the use of different proximal neighbour distances and patch membership criteria to determine how these factors altered the patches that were created. For example, how would the resulting patches change if we allow or prevent long thin shapes from joining into patches?

2) Can a spatial model be used to design large patches? The solution algorithms in most operationally-sized spatial models (as in Patchworks) are designed for "finegrained" problems, where a good solution can gradually evolve through a sequence of small incremental changes to a base problem. We have some concern these solvers might locate core areas based on their initial heuristics, and be unable to consider alternative designs because it will be trapped within a local minimum in the objective function space. Large patches are composed of a large number of pieces and are inherently coarse-grained. In order to relocate large patches to better locations the scheduler may need a significant number of transitions through an inferior space. The large number of potentially inferior transitions required would make the likelihood of success of escaping a local minimum improbable. We had not undertaken any systematic studies to confirm or dispel these suspicions, but did so as part of this study.

3) Does the spatial model reliably design large patches while maintaining other resource values?

In other words, can the spatial model fairly trade off large patch design with other spatial and aspatial management objectives? The intent was to test if the model can consistently generate equivalently valued solutions. Our concern was that each independent simulation would yield different patch configurations, and some of these configurations might have significantly different values for other resource management objectives (for example, timber harvest or operating costs). Within the Patchworks model goal programming framework some flexibility is allowed for individual sub-targets to vary, so long as the overall objective function value is minimized. Thus, we wanted to measure the range of variability among simulations, in order to determine the number required to obtain a representative sample of the solution space.

4) Can patches be used as a proxy to measure and design core areas?

We believe that patch size (the net of core area) can be used as a proxy for designing cores using a spatial model such as Patchworks, but we have not seen this attempted for large core requirements. Thus, we set out to assess the applicability of Patchworks for designing the large core areas typically required in forest management planning.

\section{The Patching Algorithm}

An important part of the patching algorithm is the definition of topological adjacency. The Patchworks model uses a proximal relationship that defines neighbours as being within a set distance of one an other (as opposed to a more rigid "touching" relationship used in conventional GIS tools).

A patch is defined as a connected set of topologically adjacent polygons, with all polygons in the set having a value for a specified patch membership criterion attribute that is greater than a specified threshold level. This essentially means that patch size and shape are determined by the topological connectivity of a polygon to its neighbours, as well as the characteristics of the polygons in terms of a specified attribute.

The value of the patch membership criteria attribute is computed independently for each polygon and planning period. The membership criterion is usually related to the structural development of a forest stand, and typically characterizes a seral stage such as "disturbed forest" or "mature forest." The patch membership attribute is dynamic, and can change over time due to management interventions or the process of forest growth and succession. As the patch membership criteria values change with time, so do the resulting patch shapes.

The model identifies patches by comparing the criteria value for each polygon to the threshold value of the patch membership attribute (typically non-zero). If the criteria value exceeds the threshold the model evaluates all the topologically connected neighbours of that polygon against the same criteria. If any of those polygons have a value greater than the threshold for the patch attribute, their neighbours are also checked and so on until all connected neighbours are found. This process is repeated for every planning period in the simulation. 
Parameters that influence the patching algorithm include:

- the distance threshold below which neighbouring polygons are considered adjacent,

- the age-dependent patch membership attribute,

- the threshold value of the patch membership attribute,

- the predefined patch size class categories.

Elements of the data set that influences the patching algorithm include the shape of the base polygons that are eligible to join into patches.

Attributes that can be set in the Patchworks solver that influence the patching algorithm include:

- the total area within each patch size class,

- the proportion of area within a size class, relative to the total area in all size classes,

- the frequency of patches within a size class,

- the average edge-to-area ratio of all patches in a size class.

Each of these attributes can be set independently for each planning period. Weighting values (used in the goal programming formulation) can also be set independently for each target and planning period.

\section{Data Set}

The Gordon Cosens Forest is located in the Boreal Forest Region in Northeastern Ontario, around the town of Kapuskasing Ontario (McPherson et al. 2008, this issue). It includes approximately 1.7 million hectares (ha) of Crown productive forest, and provides timber for the mills of Tembec, in Kapuskasing, Timmins, Hearst, Opasatika and Englehart. Tembec Forest Resources Management (Kapuskasing) is responsible for forest management planning on the management unit under the authority of the Spruce Falls Inc. sustainable forest license.

The modelling data set was derived from the data used for development of the 2005 forest management plan. As is common in planning exercises, forest stand polygons were stratified into "forest units" (forest types) for which stand dynamics (growth, yield, decline, succession, harvest and silvicultural options) were compiled by the planning team into the format used by the aspatial Strategic Forest Management Model (SFMM); (OMNR 2002). For our study, these SFMM assumptions were transcribed almost verbatim into a Patchworks data set containing 129643 forested polygons representing the entire productive forest landbase.

In addition to the forest data, we developed a roads data set that connected each stand to the mill at Kapuskasing using road segments representing existing and candidate roads. The road segments had variable haul and maintenance costs (depending on road quality and design speed), with construction costs charged on the candidate roads selected for use. The scheduling algorithm in the spatial model selects the sequence of road segments required to transport forest products from stump to mill. Accounts were used to track all transportation charges and budgets for these costs were included in the goal programming formulation.

For the purpose of this analysis, we used older conifer forest as the criterion for creating core patches. Forest polygons with a conifer-dominated forest type and a stand age of 80 years or older were given a patch membership attribute value of 1 unit per hectare. We considered that stand succession events defined in the growth and yield portion of the model would result in new stands that retained considerable struc- ture, including veteran trees. These stands, although considered young for the purposes of growth and yield, would retain their older conifer characteristics for habitat. Thus, after a succession event occurs, blocks still retained their habitat attributes. Areas either available for, or reserved from, harvest were assigned patch membership attributes.

The data set contained many riparian buffer polygons. These polygons were long and narrow, covering little area but long distances. Riparian buffer polygons are excluded from harvest, and are almost always composed of older conifer forest types in this forest. Since these polygons are long, they tend to connect large numbers of polygons via narrow strips. This results in patch shapes that are dendritic, having tentacles or large clusters connected by skinny arms. These shapes might not be considered suitable core to most forest management guideline requirements, so we produced a version of the data set that disallowed riparian buffers from joining in patches, regardless of the age or composition of the buffer.

\section{Methods}

Three sets of simulations were conducted to explore the ability of the spatial model to identify functional patches, to identify technical challenges, and the means to resolve them. For purposes of this analysis, we used late-stage conifer as the primary criterion for patch composition.

\section{Effect of patch membership criteria on patch size}

The first set of simulations assessed the effect of patch definition criteria on patch size. This assessment was conducted by systematically altering patch criteria and observing the results for a common but simple management simulation: a "no-harvest" simulation forecast over a 150 -year planning horizon with 30, 5-year planning periods.

We used a $2 \times 6$ factorial design to examine 6 topological connectivity distances (describing neighbours using separation distances of $0,10,25,50,100$ and $200 \mathrm{~m}$ ) and 2 definitions of patch membership criteria (allowing older conifer riparian buffers to join patches together or not). Each combination had only 1 replication (since only a single outcome is possible from a "no-harvest" scenario) for a total of 12 scenarios.

For each scenario, 7 different patch size classes were evaluated for several time periods over a 150 -year time horizon in the absence of harvest treatments.

The analysis used the patch size ranges given in Table 1 to test the effect of patch size criteria on resulting patch area. All classes were defined and measured in all scenarios. In addition, average patch size was compared among the 12 scenarios for each planning period.

Table 1. Late stage conifer patch size classes as defined for Patchworks scenarios in this study

\begin{tabular}{ll}
\hline Class name & Size limits (ha) \\
\hline Class 1 & $0-100$ \\
Class 2 & $100-1000$ \\
Class 3 & $1000-3000$ \\
Class 4 & $3000-7000$ \\
Class 5 & $7000-20000$ \\
Class 6 & $20000-100000$ \\
Class 7 & $>100000$ \\
\hline
\end{tabular}




\section{Creating patches while managing other objectives}

The second set of simulations assessed how well the spatial model controlled the distribution of area into various patch size classes while also factoring in other typical forestry objectives. We used a $2 \times 2$ factorial design to examine the 2 previous definitions of patch membership criteria and 2 core area size classes using a $25-\mathrm{m}$ topological search distance to define neighbours.

We used the Class 4 (3000-7000 ha) and Class 5 (7000$20000 \mathrm{ha}$ ) patch size ranges (Table 1) to test the effectiveness of the model in controlling core patch size ranges. The first range approximately represents the target size for marten core habitat, an important consideration in management planning in boreal Ontario (OMNR 1996). The second range approximately represents the target size for caribou habitat, also an important consideration in management planning in boreal Ontario (Racey et al. 1999).

The Patchworks solver makes heavy use of a random number generator to choose the sequence of changes to the management treatment schedule. By default, the same initial seed is used to prime the random number sequence, and thus Patchworks will repeatedly generate the same solution for the same set of inputs and targets. Changing the seed changes the sequence of moves used by the solver to converge on a solution. For this study 10 different numbers were chosen at random to seed the algorithm with different starting points. We hoped to observe whether the algorithm is susceptible to falling into different local minima by using different starting points.

The 40 scenarios were combinations of initial seed, size class range, and allowing or disallowing riparian area from joining patches. In each scenario the model maximized the frequency of core patches in the selected size class range while also trying to satisfy the following additional objectives (loosely derived from the 2005 management plan for the Gordon Cosens Forest, with additional operational objectives provided by Tembec planners):

- Minimum and maximum annual harvest objectives for the following species groups: spruce-pine-fir (SPF), intolerant hardwoods, other conifers,

- silviculture expenditure objectives: maximum of $\$ 4.3$ million per year and minimum of $\$ 2.4$ million per year,

- minimum and maximum cutblock opening size objectives: all cutblocks between 100 and 260 hectares in size,

- building and maintenance roading costs: maximum road maintenance expenditure of $\$ 900000$ per year and maximum road building expenditure of $\$ 550000$ per year,

- maximum hauling costs of $\$ 10$ million per year.

The weighting values used for the operational objectives were adjusted to be demanding enough to provide competition for the patch objectives, but not enough to dominate the solution. Other management objectives required by the planning process (e.g., habitat, old growth) were excluded from this analysis since they were closely correlated to the old conifer forest types used in the patch objectives.

\section{Comparing patch size (net area) to core size (gross area)}

The final set of simulations compared patch sizes (net area) measured using the spatial model to core sizes (gross area) that include interspersions within the event boundary. In particular, we were interested in the consistency between patch and core sizes and the quality of the cores in terms of the amount of interspersion.

We used 2 definitions of patch membership criteria using the Class 4 (3000-7000 ha) patch size class. Ten replications of each combination were run, for a total of 20 scenarios.

The core assessment tool in Patchworks was used to identify and measure cores for each patch $>3000$ ha. The tool utilizes a raster GIS buffer in-and-out operation to produce a shapefile containing the overlay between the event horizon and the polygons used in the spatial model. Each polygon in the resulting shapefile is identified as being part of the patch or the interspersion (see the example in Fig. 3). In this case a 10 - $\mathrm{m}$ raster size and a 200-m buffer distance were specified,

Fig. 3. The shape on the left represents the patch area [dark] recognized by the spatial model, and the shape on the right shows the output of the core assessment tool including patch areas (dark) plus the non-habitat interspersion (light).

and all blocks $>=3000$ ha were evaluated. Core areas were evaluated for each of the 20 scenarios for 3 time periods (planning periods 4,10 , and 20, representing 20,50 , and 100 years in the future).

Output from the core assessment tool was summarized in a report describing the patch area and core area for each core in each of the scenarios for each time period. The relationship between core area and patch area was calculated using a linear regression for each scenario-period combination.

\section{Results and Discussion}

The spatial model Patchworks was used to generate landscape forecasts for all of the previously described scenarios. For each scenario results included a detailed description of patch size and location for each planning period, along with indicators of sustainability typically produced by strategic wood supply models.

\section{Impact of neighbour proximity distance on patch size}

Using output from the first set of simulations (the 150-year no-harvest regime), the area-weighted average patch size was calculated for planning periods 4, 10, 20 and 30 (or 20, 50, 100 and 150 years in the future) at the 6 neighbour proximity distances. Fig. 4 shows how average patch size varied with neighbour proximity distances as the forest developed over time.

The spatial model is deterministic and contains no natural disturbance dynamics (e.g., fire or pests). Without harvest, 


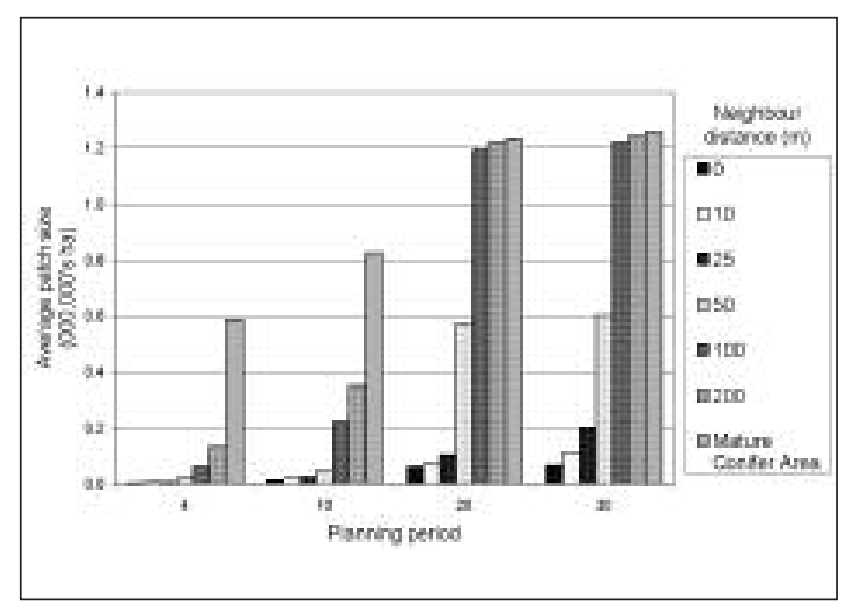

Fig. 4. The change in average patch size through time for various neighbour proximity distances using the data set that included riparian strips in patches.

the forest grows older through time. Although succession dynamics are used to characterize the change in merchantable volume characteristics, the criteria used to describe mature conifer forest have deliberately been adjusted not to reset when forecasts indicate an overstory collapse and emergence of a new canopy (we assume that this condition would actually represent significant structural diversity and therefore prime "older forest" characteristics). Ultimately, in the simple depiction of the unharvested state all conifer stands become "old." The only polygons in the data set that are not candidates to form patches would be deciduous and non-forest.

As expected, average patch size increased over time as the forest aged, and patch size also increased as the neighbour proximity distance increased. Unsurprisingly, in the later simulation periods (periods 20 and 30 , beyond 80 years of growth), the simulations with the larger proximity distances (100 and $200 \mathrm{~m}$ ) have resulted in super-patches that include almost every conifer polygon in the data set. Average patch size was very sensitive to "neighbourhood" definition, as can be seen from the small average patch sizes that occurred with short neighbour distances, and the dramatic 6-fold increase in average patch size that occurred as neighbour distance increased from 20 to $100 \mathrm{~m}$ (Fig. 4).

\section{Impact of riparian strip inclusion on patch size}

Riparian strips are typically long and narrow, and have the potential to make topological connections across widely separated areas. We anticipated that riparian strips would act as filaments that connect dispersed patches into larger topological networks. Using the output from the first experiment, we compared the area-weighted average patch sizes between the riparian-included and riparian-excluded datasets, using 0, 50 and $200 \mathrm{~m}$ neighbour proximity distances.

In all cases, the data set that allowed riparian strips to join patches resulted in larger average patch sizes than the data set where riparian strips could not join patches (see Fig. 5, 6, and 7). The most pronounced difference between average patch sizes occurred when the distance threshold was $0 \mathrm{~m}$ (only touching polygons were neighbours). At the 200-m neighbour distance, average patch sizes were very similar. The riparian strips were $60 \mathrm{~m}$ wide, so with the 0 - and $50-\mathrm{m}$ neighbour

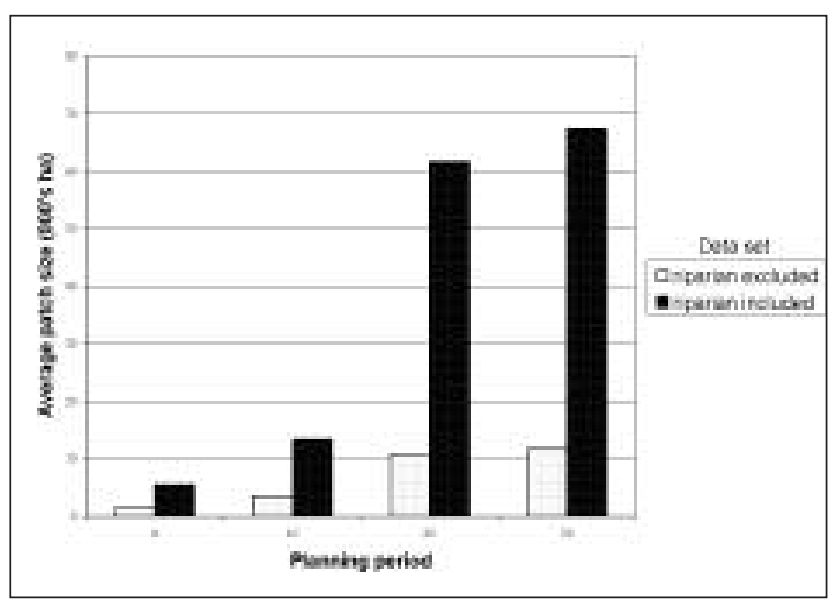

Fig. 5. A comparison of average patch size resulting from scenarios run using the riparian-included and riparian-excluded data sets at various future times, using a neighbour proximity distance of $0 \mathrm{~m}$.

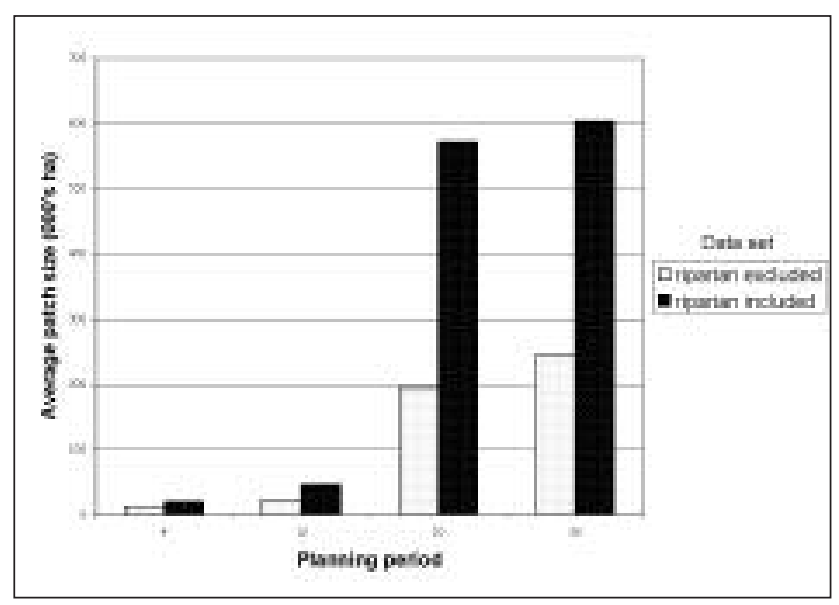

Fig. 6. A comparison of average patch size from scenarios run using the riparian-included and riparian-excluded data sets at various future times, using a neighbour proximity distance of $50 \mathrm{~m}$.

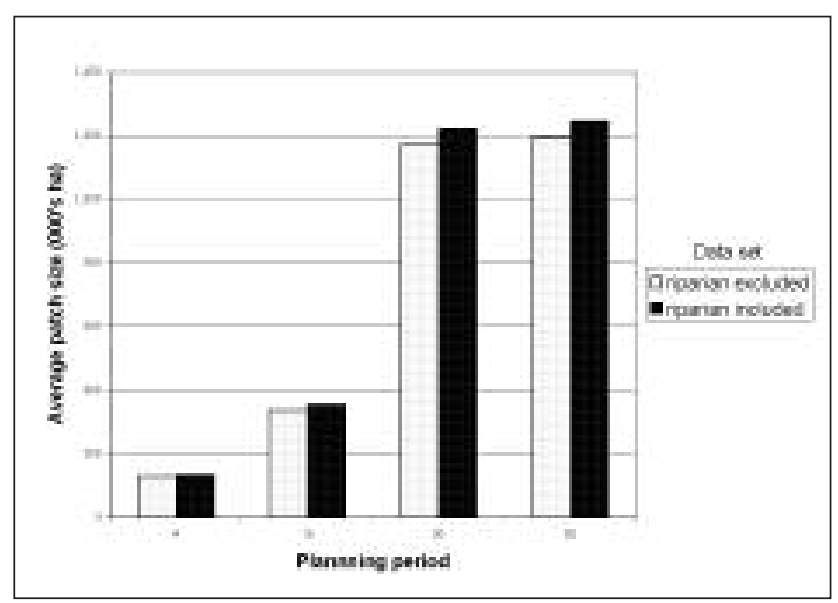

Fig. 7. A comparison of average patch size from scenarios run using the riparian-included and riparian-excluded data sets at various future times, using a neighbour proximity distance of $100 \mathrm{~m}$. 
proximity distances, the riparian strip exclusions would cause breaks to otherwise contiguous mature cover. At the 200-m separation distance the effects of "within stand" riparian exclusions is eliminated, and differences in average patch sizes can be attributed to the effect of inter-patch riparian connections. As well, at this neighbour distance the difference between average patch sizes is consistently small over all time periods. The threshold neighbour distance that is equivalent to the effect of the riparian connections seems to be $200 \mathrm{~m}$.

\section{Ability to control large patch size classes}

In the second set of simulations, the objectives were to control patch frequency in various size classes while maintaining other wood supply objectives. We conducted a "base" run with the wood supply objectives but without setting patch targets and then compared these results to a series of "controlled" scenarios that added patch targets in several size classes. One target was to achieve 100 patches in the Class 4 size category (3000-7000 ha), and the second was to achieve 50 patches in the Class 5 size category (7000-20 000 ha). Ten replications of each scenario were used to determine the average number of patches in each class over time (Fig. 8 and 9).

The base run represents the arbitrary "shotgun blast" harvest allocation that occurs in the absence of spatial control (no patch size targets). Without explicitly controlling the location of the harvest, the pattern of the residual mature conifer forest cover (and therefore the number of large harvest patches) is haphazard at best. In contrast, scenarios that include large patch size objectives successfully achieved the desired arrangement of patches, particularly in the later planning periods as more forest aged into the mature forest condition, increasing the available options. The simulations resulted in a variety of configurations, but the total area of mature conifer forest was similar among scenarios (see Fig. 10).

Stands selected for harvest were based on both the patch targets and wood supply objectives. We observed that the other targets (harvest volumes, transportation objectives, opening size limits) had less effect in the scenarios with patch size controls. In the 10 scenarios where class 4 patches were created with riparian inclusion, the drop in SPF harvest volume varied between $3.41 \%$ and $3.63 \%$. The other objectives, which were weighted lower, had greater degrees of variation. This indicates that the mutually exclusive goals were competing in the simulation runs, and the goal programming formulation traded off among multiple targets (as expected).

Intuitively, we expected it to be more difficult to achieve a simultaneous balance of wood supply and the larger patches, which was confirmed by our results (see Fig. 8 and 9). In all cases, better compliance occurred as time advanced and the amount of mature forest increased. The scenarios based on the riparian-excluded data set had fewer large patches due to reduced connectivity.

We also compared the shapes of the resulting patches, using an edge-to-area ratio metric averaged across all patches in the size class, and across all repetitions (see examples in Fig. 11 and 12). Scenarios based on the riparian-excluded data set had lower values for the average shape metric, indicating that they were more compact. This is consistent with the assumption that patches that include the mature conifer in riparian strips have dendritic shapes and are more spread out across the landscape. The shape metric was higher for the

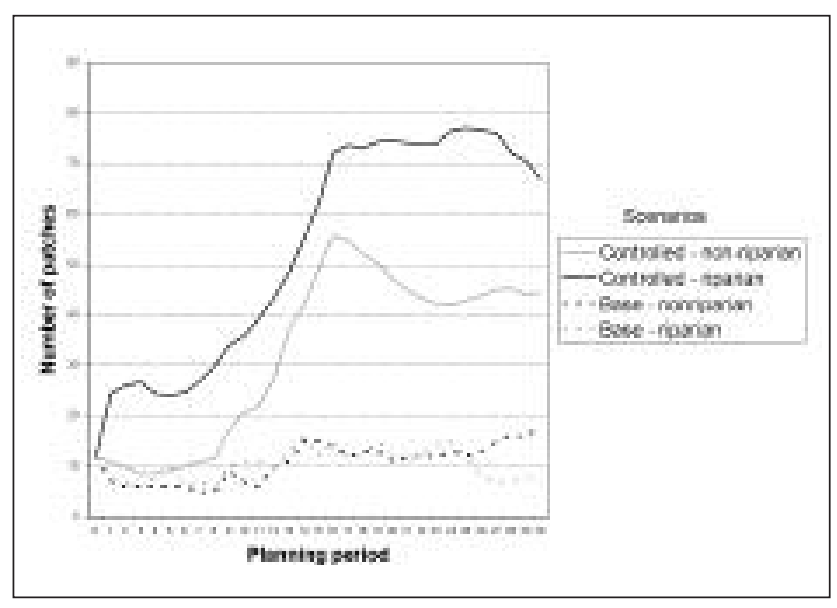

Fig. 8. The average frequency of class 4 (3000-7000 ha) patches in the base and controlled scenarios.

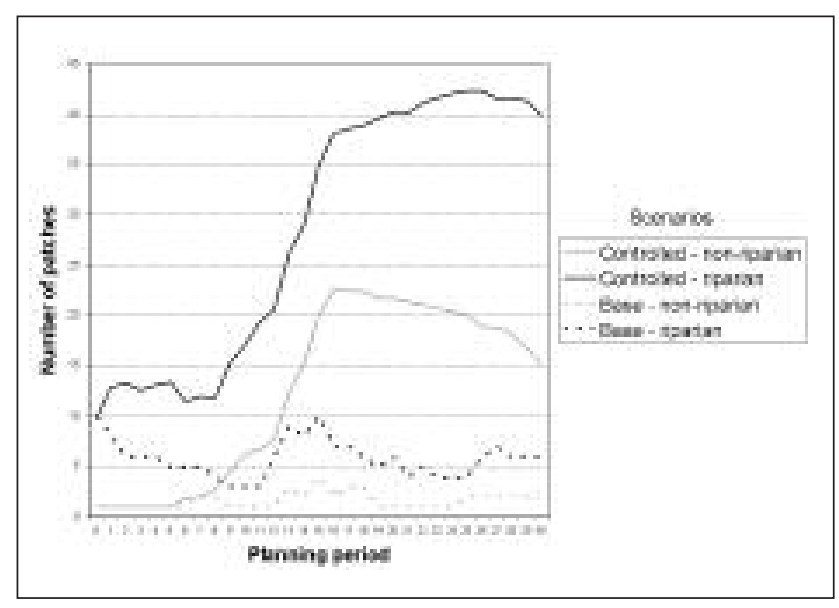

Fig. 9. The average frequency of class 5 (7000-20 000 ha) patches in the base and controlled scenarios.

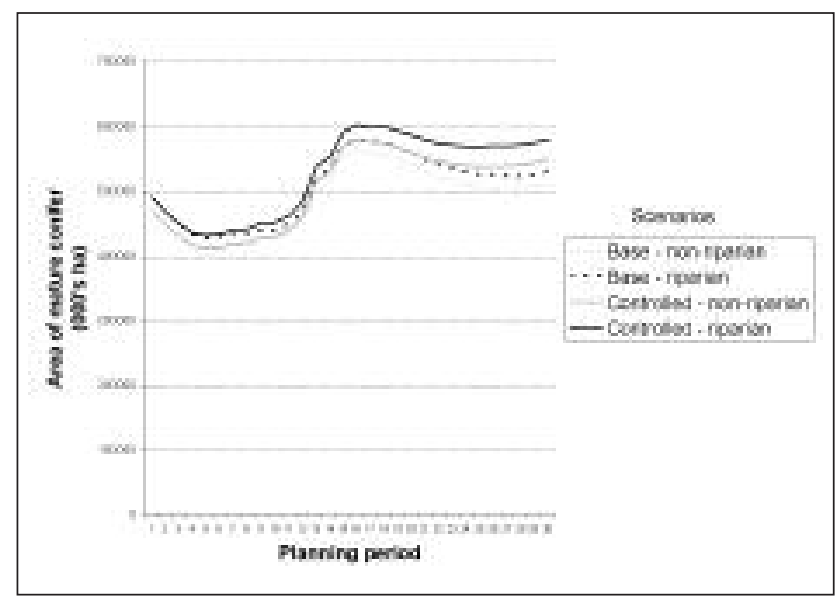

Fig. 10. Area of mature conifer forest by scenario. Regardless of the scenario, the area of mature conifer forest was similar, but the arrangement by patch size class varied considerably. 


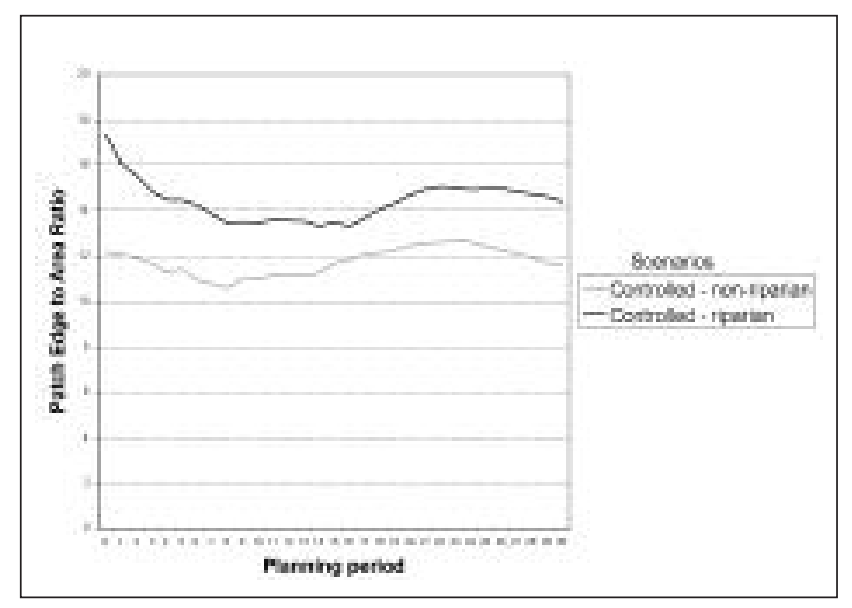

Fig. 11. The average shape of Class 4 (3000-7000 ha) patches in the riparian included and excluded scenarios.

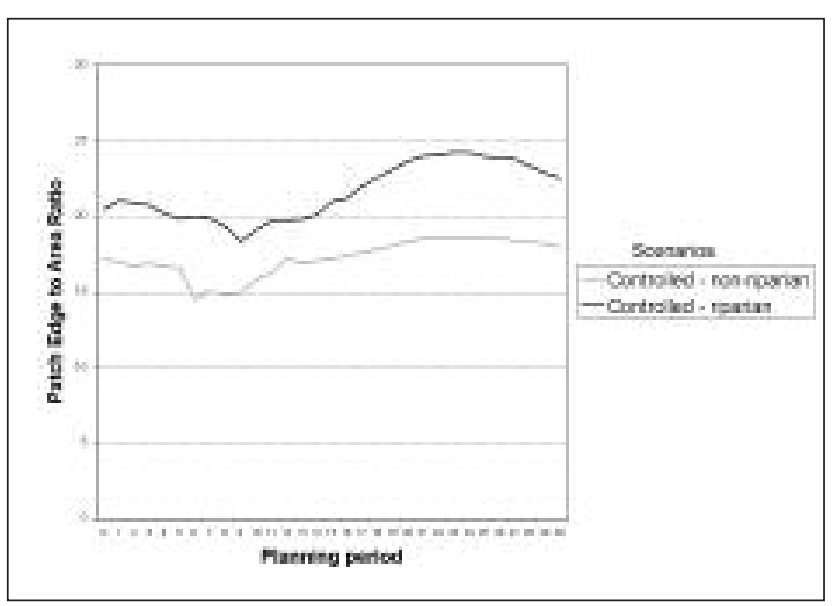

Fig. 12. The average shape of Class 5 (7000-20 000 ha) patches in the riparian included and excluded scenarios.

Class 5 patches-likely because these were opportunistically formed from thin connective corridors rather than being dense uniform contiguous areas. The shape metric was not controlled as a target within the scheduling algorithm, so the patterns are somewhat haphazard. It is likely that the edgearea ratios would have been reduced if that had been an objective, but the amount of that reduction and the effect on the other modelling objectives was not examined.

\section{Assessment of repeatability and reliability}

We compared the locations of the patches through time to determine how often the spatial model would produce patches in the same locations. Our purpose was to determine whether the spatial model would consistently select the same locations, or a variety of configurations for patches. Fig. 13 through Fig. 15 show the results of 10 repetitions of the Class 4 (3000-7000 ha) scenario using a 25-m neighbour distance for the riparian-included data set. The maps show the number of times a block contributed to the formation of a patch that was $\geq 3000$ ha. Selection frequency was evaluated for planning
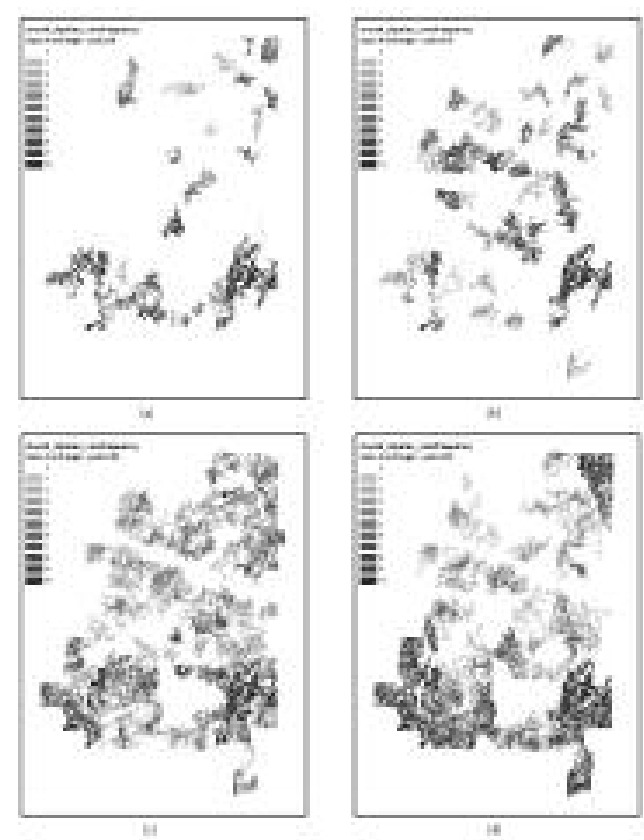

Fig. 13. Cumulative location of class 4 patches (a) in period 4 , (b) in period 10, (c) in period 20, and (d) in period 30.

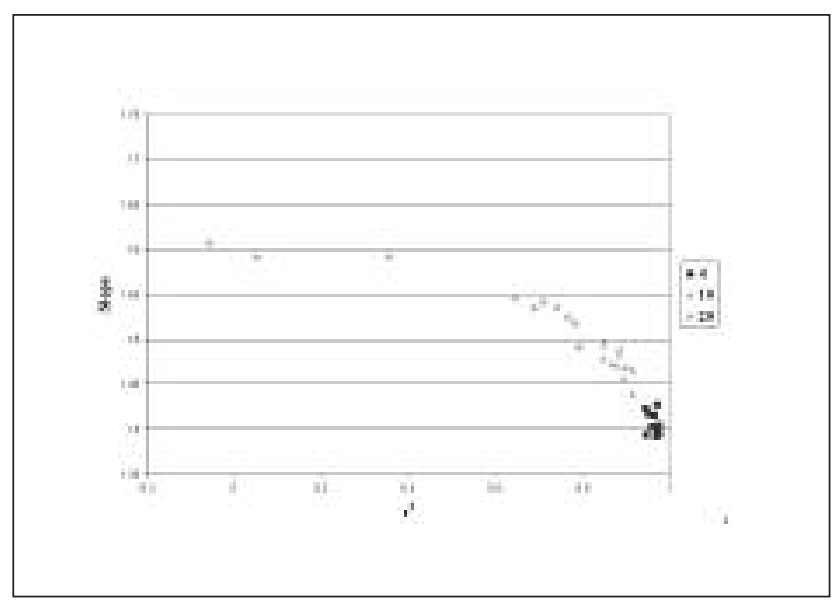

Fig. 14. Regression results for the riparian-excluded data set on all Class 4 (3000-7000 ha) and larger patches.

periods 4, 10, 20 and 30. Areas that were selected in all 10 replications were generally large parks and protected areas.

In the fourth planning period, patch locations were located quite consistently, primarily because at this stage of the simulation opportunities to vary the location of the existing older forest were limited. In periods 20 and 30 many more options were possible, and most of the harvest patches were selected in less than half the replications.

During the final planning period the spatial model consistently retained old forest in the distant corners of the manage- 


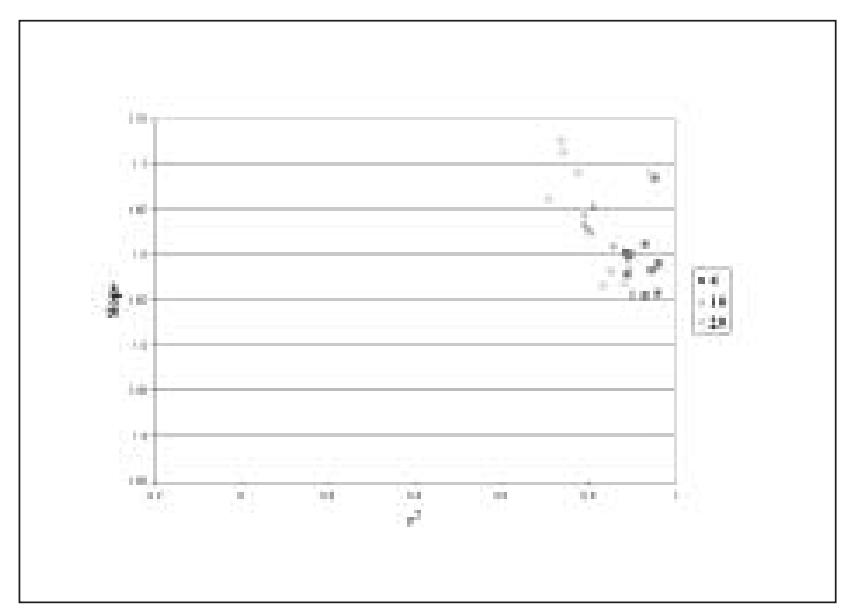

Fig. 15. Regression results for the riparian-included data set on all Class 4 (3000-7000 ha) and larger patches.

ment unit (Fig. 13d). This result is perhaps related to the targets to achieve an even flow harvest level while maintaining low transportation costs. The most expensive component of transportation costs is hauling, and the mill is near the centre of the management area. The distant wood is the most expensive, and thus the model selectively harvests this on a long rotation or defers it entirely if cheaper wood can be found elsewhere. Areas near the mill would have received intensive silviculture, so that by the end of the planning period yields on the central areas would have increased enough that the distant stands were most economically left to fulfill the old forest objectives.

When we changed the initial seed values to the random number generator the model reacted by yielding different spatial solutions and these impacted that other quantitative measures within the model. Table 2 shows the final values of the goal programming objective function for each of the 10 scenarios described above. These values varied by up to $4 \%$, similar to the previously noted variation in primary wood supply targets. This supports the idea that the solution space is large and reasonably flat, and many spatially diverse yet equivalently valued solutions are possible. Since the objective function value from the goal programming formulation is somewhat abstract from the planning goals, additional subjective criteria are required to rank which of the 10 options best suits the planning objectives.

\section{Using patch area as a proxy for core area}

We ran the core delineation tool on results from the Class 4 (3000-7000 ha) scenarios using the riparian included and excluded data sets, with a $25-\mathrm{m}$ neighbour proximity distance. Core and patch areas were compared for all Class 4 and larger patches for planning periods 4,10 , and $20(20,100$, and 150 years in the future). We then fit a straight-line relationship between patch area and core area using an intercept of 0 . A summary of slope and $\mathrm{r}^{2}$ values are shown in Fig. 14 and 15.

The riparian-excluded data set had a lower core-to-patch ratio, indicating that the riparian excluded patches were denser, and that riparian connections contributed to core areas with larger amounts of interspersion. Riparian connections joined smaller patches, and more patches were formed in the riparian-included data set.
Table 2. Objective function values for the 10 repetitions of the Class 4, 25-m separation, riparian-included scenario

\begin{tabular}{cc}
\hline Scenario & Objective function value \\
\hline 1 & $6.21 \mathrm{E} 12$ \\
2 & $6.18 \mathrm{E} 12$ \\
3 & $6.23 \mathrm{E} 12$ \\
4 & $6.04 \mathrm{E} 12$ \\
5 & $6.10 \mathrm{E} 12$ \\
6 & $6.00 \mathrm{E} 12$ \\
7 & $5.96 \mathrm{E} 12$ \\
8 & $5.99 \mathrm{E} 12$ \\
9 & $5.98 \mathrm{E} 12$ \\
10 & $6.09 \mathrm{E} 12$ \\
\hline
\end{tabular}

Patch and core area estimates were highly correlated, as shown by the $r^{2}$. The $r^{2}$ values decreased in later periods in the riparian-excluded data set, possibly due to increased fragmentation, but also because sample size increased as more patches became available in later periods. This trend was less evident for the riparian-included data set, which had more patches in the earlier periods.

\section{Conclusions}

For simplicity we frame our conclusions based on the original questions posed for this study.

1) How do neighbour proximity distance and riparian inclusion effect patch size?

Quite obviously, large distances for the neighbour proximity distance resulted in larger patches, and increased the ease of the spatial model to identify and maintain large patches on the landscape while simultaneously meeting other management goals. When we allowed riparian strips to be included in patch areas, the model identified more large patches, and the large patches had greater edge-toarea ratios and were less compact. There is no objectively correct set of patch criteria, but our experiment showed the impact of several configurations of neighbour distance and candidate criteria.

2) Can a spatial model be used to design large patches?

The spatial model successfully organized the harvest pattern to retain the older forest in patches based on the initial targets. However, our definition of a patch was simplistic, with no distinctions among the compositions of non-patch core area inclusions, which we defined simply as anything other than mature conifer. More stringent criteria are required to simulate policies that restrict the amount of disturbed forest included in mature cores. We suggest a simple 2-phase approach could be used: (i) use the methodology described here to identify candidate core areas, and (ii) exclude these areas and their interspersions from harvest for the required number of periods, thus forcing the deferrals of core areas suggested by the spatial model.

3) Does the spatial model reliably design large patches while maintaining other resource values?

We did not fully explore the interactions between the patch and aspatial goals, but our assessment is that the spatial model successfully organized the harvest pattern to retain old forest patches, while simultaneously balancing 
other wood supply and budget objectives (both spatial and aspatial). The mechanism to undertake trade-off analysis is built into the spatial model, but the size of the problem space may be too large to ever fully understand whether these tradeoffs are equitable. Spatial models are complex with a large number of system state elements, making it impossible to fully understand the rationale for resulting configurations.

4) Can patches be used as a proxy to measure and design core areas?

A consistently strong relationship between patch and core area was evident. The relationship between patch and core areas varied, and this variability changed over time, likely due to the changing abundance of older forest and large patch areas. Based on this, we conclude that patch size can be used as a proxy to design and measure core areas.

This study was limited by the few controls built into the spatial model to define patches and the lack of controls to define cores. We anticipate that planners may want more sophisticated controls, such as:

- control over the interspersion of disturbed forest within cores,

- control over the spatial distribution of patches and cores over the landscape, and

- control over the location of active roads so they do not traverse core areas.

All of these controls significantly increase the complexity of the relationships that need be tracked by the model. We are sceptical that elegant methods can be implemented to accomplish this and maintain model efficiency (in terms of time and computing resources).

A combination of the existing heuristics and informed human design, each guiding the other, may be the most effective approach for planning core areas for the foreseeable future. A useful process may be to rely on the spatial model to propose the time-series locations of patches, and then have habitat biologists and forest planners refine the boundaries of these patches into core shapes. These core shapes would then be used in a second modelling phase as deferral areas that would remove the entire core from available harvest areas. Although not guaranteed to be optimal, this process would help to locate cores through time with consideration of the dynamics of forest development, ensure that interspersions are controlled, and provide a quantitative basis for measuring and evaluating the effects of alternate designs.

\section{Acknowledgements}

The authors would like to thank Mike Malek (formerly with Tembec) and Dr. Kevin Crowe of Lakehead University for useful discussions that helped shape some of the ideas for this paper. Al Stinson of the Forestry Research Partnership provided advice, support and encouragement that made this project possible. Funding for the project was provided by the Forestry Research Partnership and the Enhanced Forest Productivity Science Program of the Forestry Futures Trust.

\section{References}

Eng, M. 1998. Spatial patterns in forested landscapes: implications for biology and forestry. In J. Voller and S. Harrison (eds.). Conservation biology principles for forested landscapes. pp. 42-75. UBC Press, Vancouver. 243 p.

[FSC] Forest Stewardship Council. 2004. National Boreal Standard. Forest Stewardship Council, Toronto, ON. 181 p.

Lockwood, C. and T. Moore. 1993. Harvest scheduling with spatial constraints: a simulated annealing approach. Can. J. of For. Res. 23: $468-478$.

Liu G., J.D. Nelson and C.W. Wardman. 2000. A target-oriented approach to forest ecosystem design - changing the rules of forest planning, Ecological Modelling 127: 269-281.

Lindenmayer, G. and J. Franklin. 2002. Conserving forest biodiversity: a comprehensive multiscaled approach. Island Press, Washington, DC. $352 \mathrm{p}$.

McPherson, S., F.W. Bell, J. Leach, P. Street and A. Stinson. 2008. Applying research for enhanced forest productivity on the Canadian Ecology Centre - Forestry Research Partnership forests. For. Chron. 84(5): 653-665.

Öhman, K. and L. Eriksson. 1998. The core area concept in forming contiguous areas for long-term forest planning Can. J. For. Res. 28: 1032-1039.

[OMNR] Ontario Ministry of Natural Resources. 1996. Forest management guidelines for the provision of marten habitat, Version 1.0. Ont. Min. of Nat. Resour., Toronto, ON. 24 p.

. 1998. A silvicultural guide for the tolerant hardwood forest in Ontario. Version 1.1. OMNR, Queen's Printer for Ontario, Toronto, ON.

2001. Forest management guide for natural disturbance pattern emulation, Version 3.1. Ont. Min. of Nat. Resour., Toronto, ON. $40 \mathrm{p}$.

. 2002. Strategic forest management model version 2.0 user guide. Queen's Printer for Ontario. Toronto, Ontario. 329 p.

2004. Forest management planning manual for Ontario's Crown forests. Queen's Printer for Ontario, Toronto. $440 \mathrm{p}$.

Racey, G., A. Harris, L. Gerrish, E. Armstrong, J. McNicol and J. Baker. 1999. Forest management guidelines for the conservation of woodland caribou: a landscape approach.. Ont. Min. of Nat. Resour., Thunder Bay, Ontario. Draft. 69 p.

Wei, Y. and H.M. Hoganson. 2007. Scheduling forest core area production using mixed integer programming. Can. J. For. Res. 37: 1924-1932.

Zipperer, W.C. 1993. Deforestation patterns and their effects on forest patches. Landscape Ecol. 8(3): 177-184. 